\title{
Eficiência da deglutição na laringectomia parcial horizontal: pensando além da segurança
}

\author{
Swallowing safety and efficiency after open \\ partial horizontal laryngectomy: \\ a videofluoroscopic study
}

\section{Ingestión de seguridad y eficiencia después de abrir laringectomía horizontal parcial: un estudio videofluoroscópico}

\author{
Andressa Silva de Freitas* \\ Guilherme Maia Zica*
}

\begin{abstract}
Pizzorni N, Schindler A, Castellari M, Fantini M, Crosetti E, Succo G. Swallowing safety and efficiency after open partial horizontal laryngectomy: a videofluoroscopic study. Cancers. 2019;11(4):549.
\end{abstract}

Nas laringectomias parciais horizontais abertas (LPHA) objetiva-se a manutenção das principais funções laríngeas (respiração, fonação e deglutição). Isso é possível devido a preservação de aproximadamente $30 \%$ do órgão com pelo menos uma unidade cricoaritenóidea funcional. Apesar disso, as alterações anatômicas pós cirúrgicos promovem sequelas crônicas irreversíveis, e a disfagia é o principal sintoma. Estudos recentes investigam os sinais oriundos dessa disfunção, ou seja, a penetração, a aspiração e os resíduos faríngeos ${ }^{1}$. Poucos trabalhos avaliam com maior profundidade os mecanismos que causam esses sinais ${ }^{2,3}$.

Os autores do artigo "Swallowing Safety and Efficiency after Open Partial Horizontal Laryn- gectomy: A Videofluoroscopic Study"2 são do Departamento Biomedico e de Ciências Clínicas "L. Sacco" da Universidade de Milão, do Serviço de Oncologia em Cabeça e Pescoço do Instituto de Câncer de Candiolo da Itália e do Departamento de Oncologia da Universidade de Turim da Itália. Neste estudo é realizada uma análise objetiva da segurança e eficiência da deglutição após a LPHA.

Dentre as LPHA, a de tipo II é definida pela ressecção de toda a cartilagem tireóide com o limite inferior representado pela borda superior do anel cricóide. Existem diferentes tipos de LPHA tipo II, diferenciados pela extensão da ressecção em supraglote e aritenóide (+ARI). Na LPHA tipo IIa o espaço pré-epiglótico e o pecíolo são seccionados

* Instituto Nacional do Câncer - Rio de Janeiro - RJ - Brasil 
de modo que a porção supra-hióidea da epiglote é poupada. Em ambos os lados os músculos constritores inferiores da faringe são dissecados, assim como os seios piriformes. A cartilagem cricóide é tracionada ao nível do osso hióide para alcançar a reconstrução laríngea por meio de uma cricohioidoepiglotopexia.

A deglutição é um complexo comportamento sensório-motor que envolve a contração e inibição coordenada da musculatura da boca, da língua, da faringe, da laringe e do esôfago bilateralmente. Em caso de falha na ocorrência de algum evento da deglutição, sua segurança e a eficiência podem ser prejudicadas. Esse mecanismo, após a LPHA tipo II, tem sido investigado na literatura ${ }^{1,3}$. A incidência de disfagia é de aproximadamente $100 \%$ imediatamente após a cirurgia, mas, geralmente, a função de deglutição se recupera espontaneamente de 3 a 6 meses de pós-operatório. No entanto, a aspiração crônica, especialmente com líquidos e resíduos pós-deglutição (em alimentos sólidos), são frequentemente detectados e aumentam o risco de pneumonia por broncoaspiração e morte.

$\mathrm{O}$ artigo ora resenhado trata-se de um estudo transversal com o objetivo de examinar as variáveis videofluoroscópicas associadas ao comprometimento da segurança e eficiência da deglutição após a LPHA tipo IIa +ARI. Foram recrutados 15 pacientes do Serviço de Otorrinolaringologia do Hospital Martini (Turim, Itália), com os critérios de inclusão: LPHA tipo IIa + ARI, queixas de deglutição, ausência de tumor evidente no último seguimento, ausência de traqueostomia, sexo masculino e tempo de acompanhamento pós cirúrgicos entre 3 a 36 meses.

A mediana da idade do grupo selecionado foi de 67 anos e do tempo de cirurgia até o último seguimento foi de 17 meses. Os estágios tumorais foram de T2N0 em 8 pacientes, T3N0 em 6 pacientes e T4N0 em 1 paciente. O movimento das aritenóides foi preservado em todos os indivíduos. Esses foram submetidos a uma avaliação videofluoroscópica padronizada da deglutição com a Unidade de Angiografia por Subtração Digital (Advantix LC Plus, General Electric) a 25 quadros/segundo, em plano de visão lateral. A oferta realizada foi de $10 \mathrm{~mL}$ de bário líquido, bário pastoso e meio biscoito umedecido com bário e avaliada por meio da escala Dynamic Imaging Grade of Swallowing Toxicity (DIGEST) publicada $2017^{4}$.
O estudo descreve que o tempo desde a cirurgia até o acompanhamento não interferiu significativamente nas comparações de segurança e eficiência da deglutição. No entanto, uma tendência para um período de acompanhamento mais longo foi registrada nos grupos mais seguros e eficientes.

Pacientes com deglutição insegura apresentaram pior fechamento neolaríngeo. Os autores afirmam que devido as alterações anatômicas oriundas da reconstrução, a ação esfincteriana é proporcionada pela aproximação da cartilagem aritenóide móvel (girando para frente e para dentro) e da epiglote (inclinando para trás). Logemann et al. (1994) afirmam que existem dois fatores críticos na recuperação da deglutição após a LPHA: o fechamento da via aérea na entrada da neolarínge (isto é, o espaço entre a cartilagem aritenóide e a base da língua) e o contato da base de língua com a parede posterior da faringe $(\mathrm{PPF})^{5}$.

Os achados demonstram que o fechamento do vestíbulo laríngeo pode ser aprimorado por meio de terapia fonoaudiológica e abordagens cirúrgicas de reabilitação. As manobras supraglótica e super-supraglótica possibilitam a maior amplitude de movimento e duração do fechamento do vestíbulo laríngeo. As abordagens cirúrgicas compreendem a injeção endoscópica de diferentes materiais na aritenóide preservada ou na face superior do anel da cricóide.

A deglutição foi considerada ineficiente em $40 \%$ da amostra do estudo. Os pacientes com deglutição ineficiente apresentaram tempo de trânsito faríngeo total mais longo, abertura lateral mais estreita do esfíncter esofágico superior (EES) e um contrato mais fraco entre a base da língua e a PPF. As pressões faríngeas dependem da ação da válvula velofaríngea, da protrusão da base da língua e da contração dos constritores da faringe. Portanto, pode-se especular que a retração incompleta da base da língua resultou em uma menor pressão faríngea, prolongando a duração do tempo total de trânsito, reduzindo a abertura lateral do EES e a elevação do osso hióide. Essa nova fisiologia aumenta o risco de resíduos pós-deglutição nos seios piriformes e valéculas.

No estudo, um aumento da viscosidade foi associado a uma maior pressão de língua, explicando assim a associação entre déficit na retração da base da língua e resíduo faríngeo com sólidos. Na terapia de deglutição, a manobra de Shaker e Mendelsohn são exercícios de fortalecimento que visam pro- 
mover uma maior abertura do EES. Além disso, a Deglutição com Esforço e a Masako melhoraram o contato entre a base da língua e a PPF. Quanto ao fechamento laríngeo, injeções de gordura têm sido propostas para a correção da perda tecidual no nível da base da língua com resultados promissores na melhora da eficiência da deglutição.

Os autores concluem que um fechamento laríngeo incompleto afeta a segurança da deglutição levando à penetração e a aspiração laringotraqueal. $\mathrm{O}$ aumento do tempo total de trânsito faríngeo, a redução da abertura lateral do esfíncter esofágico superior e a retração incompleta da base da língua causam resíduo faríngeo pós-deglutição, reduzindo a eficiência deste processo.

Os autores afirmam que a avaliação da deglutição após uma LPHA do tipo IIa + ARI deve focar na observação desses mecanismos, além da identificação de sinais de disfagia. Potencialmente, abordagens reabilitadoras e cirúrgicas podem melhorar a função da deglutição nessa população.

É possível observar que o estudo apresentou algumas limitações, o tamanho da amostra é comparável com a literatura, porém, é limitado a 15 pacientes; a distância entre a cirurgia e a avaliação videofluoroscópica é variável e a progressão a longo prazo da recuperação da função de deglutição após uma LPHA ainda é desconhecida; ausência de relatos de disfagia ou dificuldades prévias ao procedimento; avaliação videofluoroscópica com pouca clareza metodológica em relação as consistências alimentares e com apenas 25 quadros por segundo.

Os resultados do estudo demonstram que, apesar dos grandes comprometimentos laríngeos oriundos da cirurgia, é necessária a utilização de meios terapêuticos compensatórios, visando otimizar as estruturas remanescentes, como a movimentação de língua e o fechamento velofaríngeo.

O conhecimento dos mecanismos causadores de disfagia nas LPHA fornecerá base para identificar estratégias reabilitadoras e cirúrgicas direcionadas e eficazes para melhorar os resultados funcionais, reduzindo potencialmente a taxa de complicações pulmonares e o impacto da qualidade de vida. São necessários novos estudos inovadores que aprimorem a terapia fonoaudiológica nesse grupo de pacientes, para revisão de condutas e manejo de aspectos antes negligenciados.

\section{Referências}

1. Zica GM, Freitas AS, Lopes WFM, Silva BLM, Souza FGR, Freitas EQ, et al. Aspectos funcionais e epidemiológicos da deglutição na laringectomia supratraqueal extendida com traqueohiodoepiglotopexia. Distúrb Comun. 2019;31(1):87-94.

2. Pizzorni N, Schindler A, Castellari M, Fantini M, Crosetti E, Succo G. Swallowing Safety and Efficiency after Open Partial Horizontal Laryngectomy: A Videofluoroscopic Study. Cancers. 2019;11(4):549.

3. Meyer TK, Pisegna JM, Krisciunas GP, Pauloski BR, Langmore SE. Residue influences quality of life independently of penetration and aspiration in head and neck cancer survivors. Laryngoscope. 2016;127(7):1615-21.

4. Hutcheson KA, Barrow MP, Barringer DA, Knott JK, Lin HY, Weber RS, et al. Dynamic Imaging Grade of Swallowing Toxiciy (DIGEST): Scale development and validation. Cancer. 2017;123(1):62-70.

5. Logemann JA, Gibbons P, Rademaker AW, Pauloski BR, Kahrilas PJ, Bacon M, et al. Mechanisms of recovery of swallow after supraglottic laryngectomy. J Speech Lang Hear Res. 1994;37(5):965-974. 